\title{
Modeling and Simulation of Microelectrode-Retina Interactions
}

Y-12

NATIONAL SECURITY COMPLEX
November 2002

Martin Beckerman prepared by

Y-12 National Security Complex

Oak Ridge, Tennessee 37831-7622

managed by

BWXT Y-12, L.L.C.

for the

U.S. Department of Energy

under contract DE-AC-05-00OR-22800 


\section{DISCLAIMER}

This report was prepared as an account of work sponsored by an agency of the United States Government. Neither the United States Government nor any agency thereof, nor any of their employees, make any warranty, express or implied, or assume any legal liability or responsibility for the accuracy, completeness, or usefulness of any information, apparatus, product, or process disclosed, or represents that its use would not infringe privately owned rights. Reference herein to any specific commercial product, process, or service by trade name, trademark, manufacturer, or otherwise, does not necessarily constitute or imply its endorsement, recommendation, or favoring by the United States Government or any agency thereof. The views and opinions of authors expressed herein does not necessarily state or reflect those of the United States Government or any agency thereof. 


\section{Modeling and Simulation of Microelectrode-Retina Interactions}

November 2002

\section{Martin Beckerman}

Tel: 865-574-7514

Fax: 865-576-5793

Email: b3m@y12.doe.gov

prepared by

Y-12 National Security Complex

Oak Ridge, Tennessee 37831-7622

managed by

BWXT Y-12, L.L.C.

for the

U.S. Department of Energy

under contract DE-AC-05-00OR-22800 



\section{TABLE OF CONTENTS}

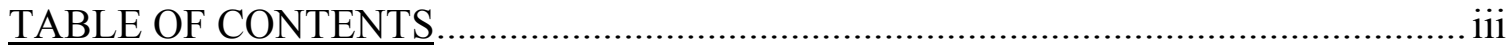

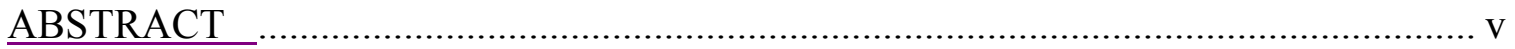

ABBREVIATIONS ……................................................................................... vii

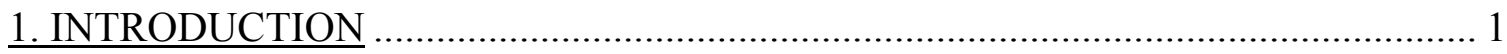

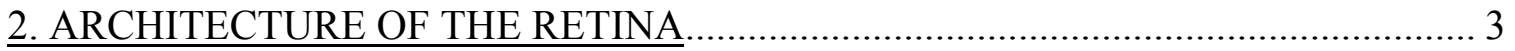

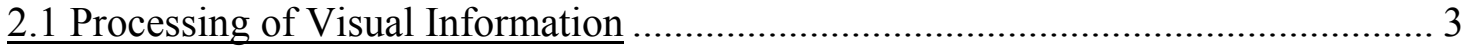

2.2 Parallel Pathways in the Visual System................................................................. 3

2.3 Local Circuits in the Normal and Disease State ................................................... 5

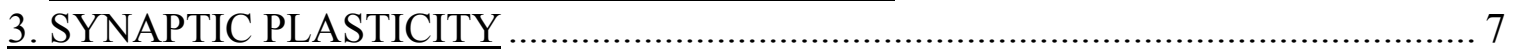

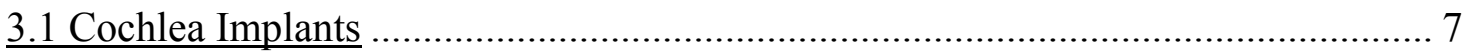

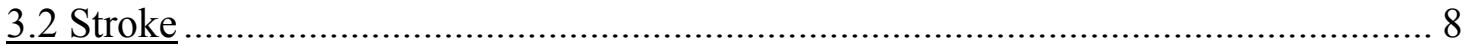

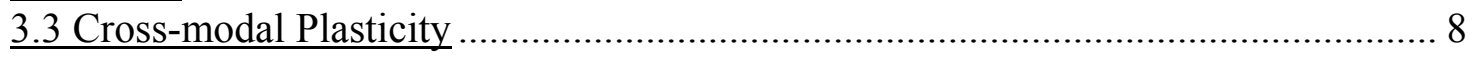

3.4 Regeneration of Peripheral, Central, and Retinal Axons ..................................... 9

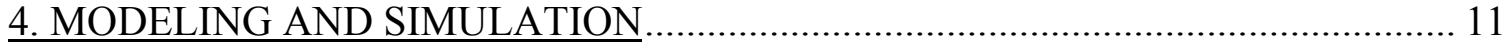

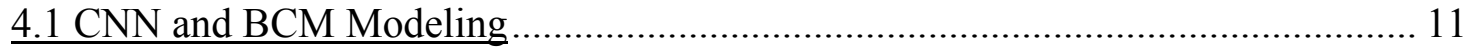

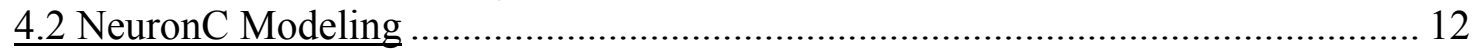

5. MICROELECTRODE-RETINA SYNAPTOGENESIS ........................................ 15

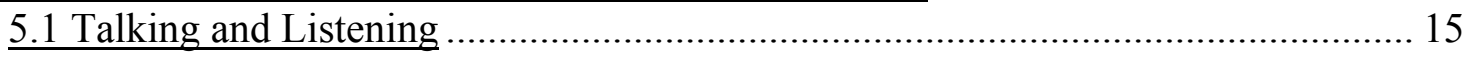

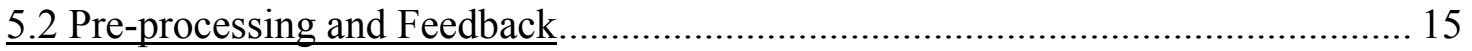

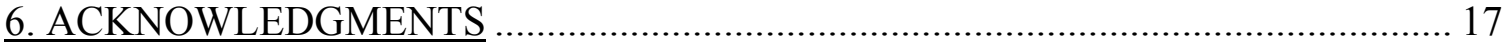

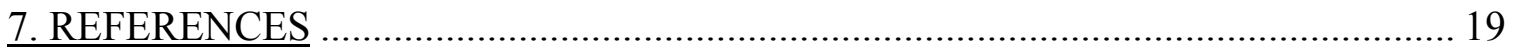


Modeling and Simulation of Microelectrode-Retina Interactions 


\begin{abstract}
The goal of the retinal prosthesis project is the development of an implantable microelectrode array that can be used to supply visually-driven electrical input to cells in the retina, bypassing nonfunctional rod and cone cells, thereby restoring vision to blind individuals. This goal will be achieved through the study of the fundamentals of electrical engineering, vision research, and biomedical engineering with the aim of acquiring the knowledge needed to engineer a high-density microelectrode-tissue hybrid sensor that will restore vision to millions of blind persons. The modeling and simulation task within this project is intended to address the question how best to stimulate, and communicate with, cells in the retina using implanted microelectrodes.
\end{abstract}


Modeling and Simulation of Microelectrode-Retina Interactions 


\[ \text { ABBREVIATIONS } \]
auditory brainstem implant
Bienenstock-Cooper-Munro
cellular neural network
central nervous system
inner plexiform layer
koniocellular
lateral geniculate nucleus
magnocellular
myelin-associated glycoprotein
microelectrode-retina interaction simulation tool
outer plexiform layer
parvocellular
peripheral nervous system


Modeling and Simulation of Microelectrode-Retina Interactions 


\section{INTRODUCTION}

The overall goal of the retinal prosthesis project is the development of an implantable microelectrode array that can be used to supply visually-driven input to cells in the retina, bypassing nonfunctional rod and cone cells, thereby restoring vision to blind individuals. There are millions of blind and visually impaired individuals. In neurodegenerative diseases, such as retinitis pigmentosa and age-related macular degeneration, functional photoreceptors are no longer present. However, much of the remaining retinal structure is present and can be used to send signals to the lateral geniculate nucleus and from there to the primary visual cortex. The implantable microelectrode device uses a camera to capture the light in place of the missing photoreceptors. It processes and converts the light into electrical signals, and then sends these visually evoked signals into the retina to the remaining cells through the microelectrodes.

Humayun and co-workers (Humayun et al. 1996) first demonstrated the feasibility of using visual input to drive the electrical stimulation of the retina. Their basic finding that the remaining cells are functional, and can be stimulated electrically, has been confirmed and supported by other groups in more recent studies (Humayun et al. 1999; Zrenner, et al. 1999) and extends earlier pioneering studies using external devices by Potts and Inoue, and others in the 1960s and 1970s. Humayun showed that retinas stimulated with arrays consisting of just a few microelectrodes could elicit a sensation of a spot of light, a phosphene.

Presently, a number of approaches are being pursued throughout the world. The various approaches can be divided into two broad classes - those that use a subretinal approach and those that implement an epiretinal one. The subretinal approach is being taken by groups in Chicago (Chow and Chow 1997; Peachey and Chow 1999; Chow, et al. 2002) and Tübingen (Zrenner 2002). Like the subretinal situation, the epiretinal strategy is being pursued by several groups. Besides the DOE-funded group, MIT-Harvard (Wyatt and Rizzo 1996; Wyatt and Rizzo 1997), Bonn (Eckmiller 1997), and Japanese (Yagi, 1999) groups are exploring epiretinal implant strategies. Perhaps the most important set of findings are those where human subjects report seeing phosphenes, which indicates that part of the retinal circuitry is present and functional in some form, and that signals are being sent from the retina via the optic nerve into the brain. Another important finding is that the implants have been shown to be safe and functional for more than one year in pigs and cats.

A major advantage of the epiretinal approach is the ability to control the conversion of light into electrical stimulation. That is, the ability exists to perform image processing at one or more levels, to optimize spatial contrast and gain, perform edge detection and other forms of feature enhancement, and evoke the best possible visual response for the given set of microelectrodes and microelectrode pulse stimulus parameters. The imageprocessing dimension is one of the focus areas of the modeling and simulation effort. Its importance will increase as the number of microelectrodes being implanted grows. 
One of the hopes of the retinal prosthesis project is that it will not require huge numbers of electrodes to produce some amount of useful vision. Whether this happens or not depends at least in part on the plasticity of the retinal and cortical circuitry. The issue of plasticity, conditions for its occurrence, and its positive and negative consequences, are other main focus areas of the study.

This report is organized as follows. The normal retina functions as a mini central nervous system with more than 50 different cell types. It uses a variety of neurotranmitters and neuroregulators. It possesses a laminar structure and utilizes an attendant set of precise circuits to carry out a number of necessary information processing tasks. A brief overview of the retina will be presented in Section 2. This will be followed in Section 3 by a discussion of synaptic plasticity and its relevance for the restoration of vision. Examples from cochlear implants, stroke, and nerve regeneration studies will be drawn upon to elucidate the key issues. The goals of the modeling and simulation efforts are then presented in Section 4.

The questions that may be asked of a model depend in large measure on the level of abstraction, and the accompanying clinical fidelity, of the models. This aspect will be addressed in the discussions presented in Sections 4 and 5. Section 5 is intended to emphasize the interplay between experiment and theory. Given the large number of unknowns associated with the deterioration of the circuitry, it is imperative that there be a dialog between the model construction and clinical treatments and laboratory experimentation. Testing, verification and validation is extremely important in situations of this sort. Finally, the idea of establishing and maintaining two-way communication between microelectrodes and neurons is advanced. Thinking about retina-microelectrode interactions as a form of synaptogenesis leads to a number of potentially useful design ideas. 


\section{ARCHITECTURE OF THE RETINA}

\subsection{Processing of Visual Information}

Light is converted to neural output by rods and cones situated in the outermost layer of the retina. The portion of the rods and cones devoted to photoreception is partitioned into an outer segment and an inner segment. The outer segment contains a set of stacked membranes enriched in rhodopsin (rods) or opsins (cones). The inner segment contains the mitochondria, ribosomes, and Golgi apparatus, while the cell nucleus sits just below the inner segment in the central part of the cell. The output, or information, conveying portion of the rods and cones sits below the nucleus and is referred to as a spherule (rods) or as a pedicle (cones). In primates, there are three kinds of cones, each maximally sensitive to a different portion of the visible spectrum - red (L) at $558 \mathrm{~nm}$, green (M) at $531 \mathrm{~nm}$ and blue (S) at $420 \mathrm{~nm}$. The cones are responsible for color (photopic) vision, while the rods supply luminance information and permit vision under dark and dim (scotopic) viewing conditions.

The feedforward portion of the information-processing stream consists of photoreceptors, bipolar cells, and retinal ganglion cells. Information from the photoreceptors is passed to the bipolar cells and from there is sent on to the retinal ganglion cells. The information being conveyed is modulated, or shaped, by the horizontal cells and by the amacrine cells. Cell bodies and synaptic contacts are interleaved in a series of layers. The spherules and pedicles of the outer nuclear layer rods and cones make synapses with bipolar cells and with horizontal cells. These synaptic triads are located in an outer plexiform layer (OPL), while the cell bodies of the horizontal, bipolar, and amacrine cells sit in a separate inner nuclear layer. Likewise, the axons from the bipolar cells make contact with amacrine and retinal ganglion cell dendrites in an inner plexiform layer (IPL). The retinal ganglion cell bodies reside in an innermost ganglion cell layer and send their axons, which form the optic nerve, on to the thalamic lateral geniculate nucleus (LGN).

\subsection{Parallel Pathways in the Visual System}

Output signals from rods and cones are combined in very particular ways by local circuits in the retina. Combinations are formed of red and green signals and of blue and red+green signals. The combinations created by the circuitry produce symmetric centersurround receptive fields for the bipolar and retinal ganglion cells. The term 'receptive field' denotes the area on the sensory periphery whose stimulation influences the activity of a downstream neuron. In the case of the visual system, the sensory periphery is the retinal surface formed by the photoreceptors, and the stimulation is the light being focused by the lens. There are two kinds of center-surround structures. One kind is of the form center-ON/surround OFF and the other takes the form center-OFF/surround-ON. In the center-ON/surround OFF case, stimulation of the central portion of the active region increases the activity of the target neuron while stimulation of the surrounding ring represses its activity. In the center-OFF/surround-ON case, the opposite response

properties are observed. There are about ten main combinations of red (L), green (M) and 
blue (S) signals. Each of these distinctive combinations is relayed by a specific kind of bipolar cell, and by a corresponding particular kind of retinal ganglion cell. These different L, M, S - bipolar-retinal ganglion cell combinations can be thought of as a set of parallel pathways into the brain, each conveying a particular piece of information about the visual scene imaged on the retina (Dacey 2000; Masland 2001).

Retinal ganglion cells can be classified according to their morphology into midget, parasol, and bistratefied types. Midget ganglion cells send their axons to parvocellular (P) cells in the LGN. The P cells send their axons to cells residing in layer 4C $\beta$. Parasol ganglion cells send their axons to magnocellular (M) cells in the LGN. The M cells send their axons to cells residing in layer $4 \mathrm{C} \alpha$. Small (bistratefied) ganglion cells send their axons to koniocellular $(\mathrm{K})$ cells in the LGN. The $\mathrm{K}$ cells send their axons to the cytochrome oxidase blobs in layer 3, and to layer 1. Different information is conveyed along each of these pathways: L-M (red/green) in the P pathway, L+M (light/dark) in the M pathway and S-(L+M) (blue/yellow) in the K pathway (Dobkins 2000; Shostak 2002).

The concept of parallel processing arose from studies of magnocellular (M) and parvocellular $(\mathrm{P})$ streams. The spatiotemporal firing properties were quite different, and in these two cell populations, it was clear that different pieces of information were being conveyed. As indicated in Table 1, M cells exhibit transient firing properties while P cells undergo sustained firing. The size of the two types of cells differs, as do their projection patterns. The $\mathrm{M}$ cells have larger receptive fields and cell bodies and are more sensitive to luminence contrast. The P cells have smaller receptive fields and cell bodies. Their luminence contrast sensitivity is also lower. Still another difference between these cell types resides in their kind of responses - linear and nonlinear. These properties have been studied mostly in the cat X and Y pathways (see Kaplan and Benardete 2001). The mapping from the cat to the monkey parallel processing streams is not entirely agreed upon but a consensus seems to be emerging-W to koniocellular, $\mathrm{X}$ to parvocellular, and Y to magnocellular (Levitt et al. 2001).

\section{Table 1. Information sent along parallel magnocellular and parvocellular pathways}

\begin{tabular}{lll}
\hline Property & Magnocellular (M) & Parvocellular (P) \\
\hline & & \\
Firing time & Transient & Sustained \\
Cell body/receptive field & Large & Small \\
Contrast sensitivity & High & Low \\
Response & Non-linear & Linear \\
& & \\
\hline
\end{tabular}

Each pathway captures and encodes specific features of the visual scene, and each has its own characteristic spatiotemporal firing pattern (Jacobs and Werblin 1998; Roska and Werblin 2001). Because of these further differences, there are about ten pathways rather than just two. The firing properties of bipolar and retinal ganglion cells can be broken down into $\mathrm{ON}$ and $\mathrm{OFF}$ centers, transient and sustained, and linear and nonlinear. 
Inhibitory feedback from horizontal and amacrine cells plays a key role in shaping the receptive fields. The laminar structure of the retina assists greatly in the formation of the pathways. Each of the distinctive bipolar cell types ramifies in a particular stratum (sublayer) of the IPL. It makes synapses with specific populations of amacrine and retinal ganglion cells, whose dendrites ramify in the same stratum.

Horizontal cells making feedback synapses on the photoreceptors are believed to be responsible for the receptive field organization of both the bipolar cells and ganglion cells. Other processes, involving amacrine cells, are thought to contribute to inhibition in transient cells and to the nonlinear component. The set of negative feedback processes utilized by both horizontal and amacrine cells is commonly referred to as lateral inhibition (Roska 2000).

The major cell populations in the mouse, cat, rabbit, and monkey retina have all been determined. The main finding (Jeon 1998) is that the major populations of horizontal, bipolar, amacrine and Müller cells is preserved across species boundaries. The fraction of each of these major cell types is fairly constant from species to species. In addition, the cell densities are preserved. The main difference among the mammalian species is the primate fovea, and in the details of the circuitry - dichromatic, trichromatic, etc. Detailed and even coarse-level information on subpopulation differences, and on differences in pharmacology and ion channel biophysics is by-and-large lacking.

\subsection{Local Circuits in the Normal and Disease State}

The retina pre-processes visual images. As just discussed, bipolar and ganglion cells possess center surround receptive fields. The firing properties of these cells can be further broken down according to the properties listed in Table 1 into ON and OFF centers, transient and sustained, and linear and nonlinear (Demb 1999; Dacey 2000; Flores-Herr 2001; Roska and Werblin 2001). The local circuitry, in particular horizontal cells making feedback synapses on the photoreceptors, is believed to be responsible for the receptive field organization of both the bipolar cells and ganglion cells. Other processes, involving amacrine cells, are believed to contribute to inhibition in transient cells and to the nonlinear component. The set of negative feedback processes utilized by both horizontal and amacrine cells is commonly referred to as lateral inhibition (Dacey 2000).

The retinal circuitry does more than just shape receptive fields. The retina is not a simple transmitter of visual information, but rather carries out a number of pre-processing operations. The neurons of the retina handle noise reduction, gain control and contract adaptation. The contrast adaptation process consists of several temporal components, each subsumed by a different portion of the retinal circuitry, and each varies somewhat among the various parallel circuits (Kim and Rieke 2001; Chandler and Chichilnisky 2001). The same may be said for properties such as nonlinearities. For instance, in the cat, $\mathrm{X}$ cells are linear while Y cells are not. The main contributor to the Y cells circuit nonlinearity is the spatial integration of temporal photoreceptor nonlinearities. That is, the wider receptive fields of the $\mathrm{Y}$ cells compared to the $\mathrm{X}$ cells are the main contributors to the nonlinearities (Hennig et al. 2002). 
That the photoreceptors are missing in patients with neurodegenerative disorders such as retinitis pigmentoasa may have profound consequences on the receptive field organization. In these situations, there are no photoreceptors for the horizontal cells to make synapses. Thus, the missing photoreceptors are most likely to be accompanied by an absence of center-surround receptive field organization of the bipolar and retinal ganglion cells. This absence may perturb the properties of the various parallel pathways. It will almost certainly affect the nominal horizontal cell mediated influences on linearity and signal duration. Interestingly, the amacrine cells may provide some measure of redundancy and, if the circuitry is sufficiently plastic, these cells might take up some of the missing functions of the horizontal cells. 


\section{SYNAPTIC PLASTICITY}

The term synaptic plasticity encompasses a broad spectrum of biochemical, electrophysiological, and anatomical changes in the efficiency of transmission across the synaptic cleft. It embraces anatomical processes such as the sprouting of new axonal and dendrite structures, the pruning or retraction and disappearance of old growths, and the changes in the membrane properties of existing growth. It includes naturally occurring cell death and other regressive processes, and it includes use-dependent changes in synaptic efficiency brought on through alterations in local intracellular circuits and in the expression patterns of membrane and cytosolic proteins. Under the heading of usedependent changes, it includes the repair and regeneration of circuits following injury and disease.

Prosthetic devices are being developed to aid in motor and sensory system reinforcement and recovery of function. These along with other therapeutic strategies have nerve stimulation and synaptic plasticity as central unifying elements.

\section{$\underline{3.1 \text { Cochlea Implants }}$}

In auditory prostheses, i.e., cochlear implants, multiple stimulating electrodes are inserted into the cochlea of the inner ear. These electrodes are situated at different locations along the basilar membrane of the cochlea. The basilar membrane is tonotopically arranged; high frequency sounds activate hair cells at the base, and low frequency sounds activate hair cells near the apex. Sounds reaching the implant are analyzed by a microprocessor. Electrical signals are generated that represent the sounds from each frequency region. These signals are then fed to electrodes placed at the corresponding tonotopic locations (Rauschecker and Shannon 2002). The effectiveness of the implants increases in time. Synaptic plasticity of the circuitry enables the patient to relearn how to hear with the auditory prosthesis.

One of the most interesting developments in this arena from the design viewpoint is the emergence of auditory brainstem implants (ABIs). These are intended to work in patients who cannot be helped by conventional cochlea implants because of the deep location of the auditory damage. Patients with neurofibromatosis type 2 (NF2) receive implants containing microelectrodes that penetrate the ventral cochlear nucleus (McCreery 1998). The electrodes are of differing lengths. The variable length array design allows for the penetration of the various layers of the nucleus, transmitting sounds at different pitches. The objective of this design strategy is to get past the poor patient responses elicited by earlier NBIs whose electrodes are all of the same length and where the devices sit on top of the nucleus rather than penetrate its layers.

An issue that emerges in the development of ABIs is how to elicit meaningful auditory sensations in patients. This issue arises since the information processing steps carried out by the upstream neurons are being bypassed by the implant. Considerations must be given to the form of the electrical stimulation vis-à-vis information processing that allows 
for meaningful interpretation of the signals by higher order neurons situated in the acoustic cortex (Rauschecker and Shannon, 2002).

\subsection{Stroke}

One of the most significant sets of findings on strokes is that neural plasticity plays an important role in recovery. The plasticity takes the form of regeneration of portions of circuitry. Mimicking the processes takes place during development; neurite outgrowth, growth cone pathfinding, and synapogenesis takes place resulting in new sets of neural connections that underlie the restoration of function in the subject (Stroemer 1995; Xerri 1998; Johansson 2000). The parallelism of stroke recovery with nerve regeneration in the peripheral nervous system has been studied in the somatosensory cortex in a variety of mammals. Injuries and disease states are mimicked in these studies through surgical manipulation. The manipulations include the amputation of digits and nerve crush. Sprouting and other developmentally associated processes are observed to follow these injuries and drive the recovery (Jones 1998; Jain 2000).

There are positive implications for the retinal prosthesis project from the stroke data. The reintroduction of visual input into the retina should trigger a recovery phase. Over timeperhaps many months in duration - a portion of the retina may reconstitute itself. Receptors and other components of the plasma membrane that were down regulated may become upregulated, and perhaps more dramatic, gene-expression-driven plastic processes may occur as well as stimulatory signals are reintroduced. Some of the circuitry that may have atrophied will now have a chance to undergo functional recovery in a way resembling acoustic, somatosensory, and stroke recovery. One of the long-range goals of the modeling and simulation effort is to study how the help this recovery phase.

\subsection{Cross-modal Plasticity}

A form of neural reorganization, known as cross-modal plasticity, might be thought to work against the success of a retinal implant. In cross-modal plasticity, regions of the cortex that no longer receive useful input from one sensory modality are invaded and taken over by another modality. Thus, a person who is deaf from birth may no longer have a complete functional acoustic cortex to process acoustic signals. In these cases, cochlea implants do little towards restoring hearing. The same might be expected of retinal implants. However, the appropriation of a region of cortex by a competing modality is mostly encountered in subjects deaf or blind from birth or from early childhood. It is not expected to be a significant impediment in adults either to cochlear implants (Lee 2001) or to retinal implants (Cohen 1997).

A combination of intrinsic and extrinsic factors contributes to cross modal plasticity. Some of the features that characterize visual, auditory, and somatosensory cortices are laid down through developmental programming and are thus intrinsic to the cortex (Miyashita-Lin 1999). Other properties are regulated by the nature of the inputs being related in through the thalamic nuclei and are therefore extrinsic to the cortex. Particularly dramatic examples of structures characteristic of visual cortex can be induced 
in the auditory cortex by redirecting visual input to that region (Sharma 2000). These structures are functional; they are accompanied by visual behavioral modifications (von Melchner 2000). In a recent study in developmentally blind mammals, cross-modal plasticity was shown to have strong effects on the allocation of space within the primary visual cortex, area 17, to the various competing sensory input modalities (Kahn and Krubitzer 2002). One implication from the various studies on cross-modal plasticity is that prosthetic devices are likely to provide little or no benefit to individuals deaf or blind from birth or early childhood.

\subsection{Regeneration of Peripheral, Central, and Retinal Axons}

Peripheral nerves have the ability to regenerate following injury. The rebuilding of the neural circuitry is a complex process that contains many of the same steps as required in initial circuit formation. Axon regeneration in the central nervous system (CNS) differs from that occurring in the peripheral nervous system (PNS). In the CNS of adult vertebrates, plasticity is suppressed by signals from neighboring cells and by intracellular signals.

In adult mammals, axons in the peripheral nervous system (limbs) are able to regenerate themselves, while axons in the central nervous system (brain, optic nerve and spinal cord) are remarkably resistant to new growth after injury. New axonal growth in the central nervous system appears to be restricted to prenatal life, and the process is rapidly down regulated after birth. One of the places this process has been studied is in the retina where experiments have been performed (Goldberg 2002) on the axons of retinal ganglion cells. Recall from the last section that, in the retina, amacrine cells form synapses with ganglion cells. The amacrine cells send signals to the ganglion cells that instruct them to stop growing axons and start making dendrites. Axon outgrowth predominates shortly before birth, but shortly after birth this growth stage ceases and dendritic growth picks up.

Axons of spinal cord neurons of adult mammals do not regenerate for reasons similar to those seen in the retina. Like the retinal ganglion cells, a lack of stimulatory signals plus the presence of inhibitory cues prevent regeneration. Several steps are involved in the recovery process. The axon must grow out from the cell body and travel a long distance before reaching its cellular target with which it must then establish a synapse.

The finding that axons belonging to the central nervous system do not regenerate after injury in adults whereas axons of the peripheral nervous system readily regenerate themselves following injury is quite general. A major factor contributing to the difference between responses in the CNS and PNS has to do with signaling from myelin. Axons, particularly those that must transmit action potentials over long distances, are insulated by myelin ( $80 \%$ lipid, $20 \%$ protein). In the PNS, Schwann cells encircle the axons forming a myelin sheath. In CNS axons, a class of glial cells, called oligodendrocytes, forms the myelin sheath about the axons. Schwann cells support axonal regeneration but oligodendrocytes inhibit it. Several inhibitory regulators have been identified in CNS myelin. One of these is myelin-associated glycoprotein (MAG) and another is Nogo. 
Several strategies have been devised to overcome this form of inhibition in order to develop therapies for spinal cord injuries. One strategy involves using an antibody against Nogo (Chen 2000). A second strategy is to intercede at a place downstream from the initial signal, in this case impeding a Rho GTPase stimulated by MAG (Lehmann 1999).

The retina is part of the CNS, not the PNS. However, the inhibitory effects of myelin upon long CNS axons are not present in the unmyelinated thin and short axons of the horizontal and bipolar cells and in the largely absent axons of the amacrine cells. Supporting glial cells should be present and the regenerative ability vis-à-vis sprouting and rebuilding axonal and dendritic contacts might be appreciable. The striking difference between vertebrate resistance to regeneration in the CNS and the extreme plasticity and regenerative ability in lower vertebrates is attributed to myelination. Since this is absent in the retina then perhaps so is the inhibition.

There is evidence that the retina can be quite plastic in its responses to injury and disease. Morphometric data has been acquired on how well cells in the inner retina survive in the presence of retinitis pigmentosa. While a profound cell loss takes place in the outer layer, there is a reduced, although still considerable, loss in both the inner nuclear layer and in the ganglion cell layer (Santos 1997; Humayun 1999). More importantly, cells undergo extensive sprouting in the presence of photoreceptor loss. In a mouse model of retinitis pigmentosa, extensive sprouting of horizontal cells was observed. These activities were accompanied by the progressive atrophy of dendrites of cone bipolar cells and underdevelopment of dendrites of rod bipolar cells (Strettoi 2002). In another study, sprouting by amacrine cells was observed (Fariss 2000). This may ultimately be a "good news, bad news" story. The presence of plasticity means that appreciable rebuilding of the circuitry might take place when the remaining cells are electrically stimulated, and over time useful vision may occur as the cells in the retina adjust to the receipt of input signals once again. 


\section{MODELING AND SIMULATION}

Several different kinds of models exist that can be used to explore how the retina works, how it may interact with electrical stimulation, and what kinds of signals may be sent on to the visual cortex through the LGN, the thalamic relay nucleus. The various models can be arranged in a hierarchy extending from finely detailed compartmental models at one end to coarse-grained neural circuit models at the other end of the hierarchy.

In the neural simulator based models, one constructs a molecular level description of a neuron in terms of distinct compartments for the soma, dendritic tree and axons. One specifies the biophysical and pharmacological properties of each compartment such as the numbers and distributions of ligand and voltage gated ion channels, metabotropic receptors, and neurotransmitter release properties. The emphasis and goals when constructing multi-neuron circuit models is on the circuitry itself and less so on the detailed biophysics of the individual neurons. The goal is to simulate sufficiently large expanses of neural tissue, answering questions on, for example, how synaptic plasticity develops, how it influences receptive field formation and information processing properties such as edge orientation selectivity, motion selectivity, spatial frequency selectivity, and ocular dominance.

There are specific questions that can be asked of the models at each level of abstraction. If the questions are well matched to the model, the answers can be useful not only in answering the questions but also in pointing out missing information and in raising issues that challenge the technology and therapeutic strategy.

\section{$\underline{4.1 \mathrm{CNN} \text { and BCM Modeling }}$}

There are a number of ways of modeling and simulating neural circuits. A new approach to studying circuit behavior is to construct a cellular neural network (CNN) model of the circuitry. The conceptual basis for $\mathrm{CNN}$ modeling is the cellular neural networks sometimes called Chua circuits. The core ideas behind Chua circuits were developed over the past decade or so at Berkeley and Budapest (Chua and Yang 1988; Chua and Roska 1993; Roska and Chua 1993). The modeling tool, written in a specialized CNN programming language, is being converted into a standard C-code form that can be used to model network behavior (Bálya 2002, private communication).

One of the questions that must be answered if the spatial resolution is to be determined is what is the spatial extent of the activity evoked through an electrode. That is, how large a population of cells will have their membranes depolarized beyond the threshold for activity. This question is especially relevant given that the bipolar cells being targeted in the project are second order neurons that supply graded responses to changes in membrane potential. Retinal ganglion cells, in contrast, are spiking neurons and have a pronounced threshold. The issue of fan-out arises here in a strong way. The electrodes will stimulate multiple bipolar cells, and these in turn will stimulate amacrines that will make gap junctions and form an extensive network of lateral connections. The amacrines 
may spread the area being influenced by an electrode or as mentioned earlier compress it through lateral inhibition.

One question that can be asked is since an electrode replaces visual stimulation of the retina, what is the size of the receptive field of retinal ganglion cells situated at different locations in the retina. In this situation, the bipolars comprise the sensory periphery, and electrical stimulation operates instead of light stimulation. The converse question can be asked as well. That is, how big a fan-out will occur-what is the size of the image at the retinal ganglion cell layer compared to the object, the electrode. Once these complementary questions are answered, one can address the questions of how to optimize contrast and other properties of the visual image conveyed by the array of electrodes.

As part of a series of studies on the rabbit retina, CNN parameters for the different kinds of parallel pathways were deduced (Roska and Werblin 2001). Parameters of interest included time constant, coupling strength between neurons (symmetric and isotropic), density (distance between adjacent neurons), and transmitter output function (voltage to transmitter release). Five different types of retinal ganglion cells were modeled: ON bistratefied, ON sluggish, ON brisk TrN, OFF brisk Tr, and OFF brisk L. The output patterns from the simulations qualitatively matches the observed rabbit spiking patterns from the retinal ganglion cells, and the excitation and inhibition patterns from the bipolar and amacrine cells (Bálya 2002).

An even more abstract approach can be taken using the Bienenstock-Cooper-Munro (BCM) formalism (Bienenstock et al. 1982). This well-established modeling approach has been used over the past 20 years to explore the issue of synaptic plasticity in CNS neural circuits. Results of simulation compare well with a body of experimental data on synaptic plasticity in sensory and motor systems. In implementing this model on the ORNL supercomputers, Shouval et al. 2000 was able to explore synaptic plasticity in the primary visual cortex. In Shouval et al. 2000, the goal was to model layers 2, 3, and 4 of a $3.5 \times 3.5 \mathrm{~mm}$ slice of $\mathrm{V} 1$ cortex receiving feedforward input from the retina through the LGN, and including a network of long-ranging horizontal (lateral) connections. The cortical network was stimulated by a series of digitized natural scenes, and the evolution of the connections over time was followed. The long-ranging horizontal connections were shown to form a scaffold upon which plastic changes were implemented. An appropriate combination of $\mathrm{CNN}$ and $\mathrm{BCM}$ modeling techniques may provide useful guidance on plastic and regenerative properties of the retinal-thalamic-cortical circuitry.

\subsection{NeuronC Modeling}

There are a number of questions that require a more detailed and fine-grained modeling and simulation approach. These include questions of a geometric nature regarding the efficacy of various electrode geometries and orientations with respect to various portions of the neural surface-axons, soma and dendrites. For example, a shortcoming of stimulating the retinal ganglion cells is the loss of spatial resolution resulting from exciting axons. One hypothesizes that by orienting the microelectrodes one can selectively excite neurons oriented in a particular way in specific lamina. Similarly, the 
pulse timings can be used to selectively excite different types of retinal neurons. These important findings might be greatly strengthened by a careful examination of neural simulation protocols (pulse shape and timing, and microelectrode orientation) using a fine-grained simulation approach as applied to a spectrum of cell types.

Researchers in the field have developed a number of neural simulators. Two examples widely used in the research community are Neuron (Hines 1989) and NeuronC (Smith 1992). The NeuronC simulator is specifically designed to handle multineuron circuits. It was developed by vision research in the Sterling and Smith Laboratories at the University of Pennsylvania for the purpose of understanding the organization and function of the retina. One can adapt the simulator for use in a microelectrode-retina interaction simulation tool (MIST).

The MIST can be used to explore not only protocol questions but also questions that address the deterioration in the circuitry due to retinal disease. The precise set of parallel pathways seen in normal mammals is likely to be severely perturbed and compromised. For example, as discussed above, the receptive field structure is likely to be perturbed by the loss of lateral inhibition from the horizontal cells. One way to proceed is to start with a broad set of neural response types and explore how well one can communicate through electrical stimulation with each of the cell types. Once that is done, one can probe what might occur if the normal circuitry and channel/receptor pharmacology is disturbed.

One of the parameters that plays an important role in determining spatial resolution is the electrical conductivity. Some insights into the appropriate values for the electrical conductivity are supplied by studies of electrical feedback from horizontal cells to cones (Kraaij 2000; Kamermans 2001).

The pharmacology of the neurons, and the circuitry within the IPL, are two key determinants of the signals sent out through the optic nerve from the retinal ganglion cells. Gap junctions must be included to treat the horizontal networking capabilities of the amacrines. The pharmacology and electrophysiology of the gap junctions formed between cone bipolar cells and AII amacrines have been examined (see Hartveit 1999) and this and other amacrine circuit studies serves a a starting points for the constriction of MIST models. Amacrine-amacrine and amacrine-ganglion cell couplings are all mediated to a large extent by heterologous, but in some cases homologous, couplings (Mills and Massey 2000; Vaney and Weiler 2000). 
Modeling and Simulation of Microelectrode-Retina Interactions 


\section{MICROELECTRODE-RETINA SYNAPTOGENESIS}

Microelectrodes are intended to replace missing photoreceptors and their signaling. In normal individuals, signals are sent across synapses from the pre-synaptic photoreceptors to the post-synaptic bipolar cells. Horizontal cells participate in the circuitry and play an important role in establishing the receptive field properties of the bipolar and retinal ganglion cells. In the case of blind individuals, electrical currents from microelectrodes replace neurotransmitter release from the photoreceptor, and also perhaps from the horizontal cells, too. The remaining circuitry consists of bipolar cells, amacrines and retinal ganglion cells. There are considerable uncertainties in the mix of receptors and ion channels upregulated and expressed on the surfaces in these cell populations, because of the absence of sustained signaling. The ON-OFF receptive fields properties, and perhaps other properties as well, are likely to be profoundly altered. Thus, it is unclear experimentally how to best establish and maintain communication with these cells, and unclear theoretically how to proceed with testing, verifying, and validating the model construction.

\section{$\underline{5.1 \text { Talking and Listening }}$}

One way to approach this issue is by establishing a feedback path from the cells being electrically stimulated to the electronics supplying the signals. That is, one should establish some sort of dialog between the stimulating electrodes and the cells being spoken to. If the latencies and timings are such that a single electrode cannot serve both as transmitter and as receiver than these functions can be distributed among two or more electrodes. One of the goals of the two-way communication, with or without intermediaries, is to confirm and improve on the communication. One can fine tune pulse shape, form, and timing-duration and inter-pulse interval, identifying pulse characteristics that best elicit a good response from the bipolar cells. Another goal of the two-way communication is to determine experimentally how a signal spreads among the cells. Using a single signaling electrode and multiple listening electrodes, a profile can be built of how the signals spread among cells. These signaling profiles can be compared to the model predictions for the different stereotypic cell types and circuits.

To facilitate the process of microelectrode-retina synaptogenesis, designs of the array might be considered that allow for different length microelectrodes, like the ABIs. These would create a three-dimensional geometry that might increase the chances for good synaptic contacts and for listening in on the conversation.

\subsection{Pre-processing and Feedback}

One of the advantages of the epiretinal approach over the subretinal one is the capability of processing the visual data stream. In arrays constructed with only a few microelectrodes, there is little benefit from this capability, and little more than a spot of light may be produced. As the size of the arrays is increased, more attention to contrast

and information processing can be paid. This activity is distinct from optimization and 
tuning of the electrical pulses, and refers to how the visual scene encoded as pixel intensities in a two-dimensional array is converted in currents flowing in a far smaller number of microelectrodes. It makes possible addressing a host of questions dealing with recreation of receptive fields, with adding or taking away noise, with gain, dynamic range, and contrast maximization. These issues are not abstractions, but rather are addressed by every normal retina as it processes visual scenes. Here as in the previous discussion, feedback and experimentation with different signal processing strategies can help drive the effort, and when combined with pre-processing can increase the chances for success in the endeavor. 


\section{ACKNOWLEDGMENTS}

The author wishes to thank Professor Robert G. Smith for numerous stimulating and highly informative discussions. The author also wishes to thank Drs. Mark Humayun and Jim Weiland for helpful discussions. The author wishes to acknowledge the support and project leadership of Dr. Elias Greenbaum and the enthusiastic support of Drs. Dean Cole and Mike Viola at the U.S. Department of Energy. 
Modeling and Simulation of Microelectrode-Retina Interactions 


\section{REFERENCES}

Bálya D, et al. 2002 A CNN framework for modeling parallel processing in a mammalian retina. Int. J. Circ. Theor. Appl. 30: 363-393.

Bienenstock EL, Cooper LN and Munro PW 1982 Theory for the development of neuron selectivity: orientation specificity and binocular interaction in visual cortex. J. Neurosci. 2: $32-48$.

Chandler D and Chichilnisky EJ 2001 Adaptation to temporal contrast in primate and salamander retina. J. Neurosci. 21: 9904-9916.

Chen MS, et al. 2000 Nogo-A is a myelin-associated neurite outgrowth inhibitor and an antigen for monoclonal antibody IN-1. Nature 403: 434-439.

Chow AY and Chow VY 1997 Subretinal electrical stimulation of the rabbit retina. Neurosci. Lett. 225: 13-16.

Chow AY, et al. 2002 Subretinal implantation of semi-conductor-based photodiodes: durability of novel implant designs. J. Rehab. Res. Dev. 39: 313-322.

Chua LO and Roska T 1993 The CNN paradigm. IEEE Trans. Circuits and Systems I 40: 147-156.

Chua LO and Yang L 1988 Cellular neural networks - theory. IEEE Trans. Circuits Systems 35: $1257-1272$.

Cohen LG, et al. 1997 Functional relevance of cross-modal plasticity in blind humans. Nature 389: 180-183.

Dacey D 2000 Center surround receptive field structure of cone bipolar cells in primate retina. Vis. Res. 40: 1801-1811.

Dacey DM 2000 Parallel pathways for spectral coding in primate retina. Annu. Rev. Neurosci. 23: 743-775.

Demb JB, et al. 1999 Functional circuitry of the retinal ganglion cell's nonlinear receptive field, J. Neurosci. 19: 9756-9767.

Dobkins KR 2000 Moving colors in the lime light. Neuron 25: 15-18.

Eckmiller R, et al. 1997 Learning retina implants with epiretinal contact. Ophthalmic. Res. 29: 281-289. 
Fariss RN, Li ZY and Milam AH 2000 Abnormalities in rod photoreceptors, amacrine cells, and horizontal cells in human retinas with retinitis pigmentosa. Am. J. Ophthalmol. 129: 215-223.

Flores-Herr N, Protti DA and Wässle H 2001 Synapt5oc currents generating the inhibitory surround of ganglion cells in the mammalian retina. J. Neurosci. 21: 4852-4863.

Goldberg JL, et al. 2002 Amacrine-signaled loss of intrinsic axon growth ability by retinal ganglion cells. Science 296 1860-1864.

Greenberg RJ, et al. 1998 Different cellular time-constants allow selective electrical stimulation of retinal neurons. Invest. Ophthalmol. Vis. Sci. 39: S309 (Abstract).

Hartveit E 1999 reciprocal synaptic interactions between rod bipolar cells and amacrine cells in the rat retina. J. Neurophysiol. 81: 2923-2936.

Hennig MH, Funke K and Wörgötter F 2002 The influence of different retinal sub-circuits on the nonlinearity of ganglion cell behavior. J. Neurosci. 22: 8726-8738.

Hines M 1989 A program for simulation of nerve equations with branching geometries. Intern. J. Biomed. Comput. 24: 55-68.

Humayun MS, et al. 1996 Visual perception elicited by electrical stimulation of retina in blind humans. Arch. Ophthal. 114: 40-46.

Humayum MS, et al. 1999 Morphometric analysis of the extramacular retina from postmortem eyes with retinitis pigmentosa. Invest. Ophthalmol. Vis. Sci. 40: 143-148.

Humayun MS, et al. 1999 Pattern electrical stimulation of the human retina. Vis. Res. 39: 2569-2576.

Jacobs AL and Werblin FS 1998 Spatiotemporal patterns at the retinal output. $J$. Neurophysiol. 80: 447-451.

Jain N, et al. 2000 Growth of new brainstem connections in adult monkeys with massive sensory loss. Proc. Natl. Acad. Sci. USA 97: 5546-5550.

Jeon CJ, Strettoi E and Masland RH 1998 The major cell populations of the mouse retina. $J$. Neurosci. 18: 8936-8946.

Johansson BB 2000 Brain plasticity and stroke rehabilitation. Stroke 31: 232-230.

Jones EG and Pons TP 1998 Thalamic and brainstem contributions to large-scale plasticity of primate somatosensory cortex. Science 282: 1121-1125. 
Kahn DM and Krubitzer L 2002 Massive cross-modal cortical plasticity and the emergence of a new cortical area in developmentally blind mammals. Proc. Natl. Acad. Sci. USA 99: 11429-11434.

Kaplan E and Benardete E 2001 The dynamics of primate retinal ganglion cells. Prog. Brain Res. 134: 1-18.

Kim KJ and Rieke F 2001 Temporal contrast adaptation in the input and output signals of salamander retinal ganglion cells. J. Neurosci. 21: 287-299.

Lee DS, et al. 2001 Cross-modal plasticity and cochlear implants. Nature 409: 149-150.

Lehmann M, et al. 1999 Inactivation of Rho signaling pathway promotes CNS axon regeneration. J. Neurosci. 19: 7537-7547.

Levitt JB, et al. 2001 Visual response properties of neurons in the LGN or normally reared and visually deprived macaque monkeys. J. Neurophysiol. 85: 2111-2129.

Masland RH 2001 The fundamental plan of the retina. Nat. Neurosci. 4: 877-886.

McCreery DB, et al. 1998 Accessing the tonotopic organization of the ventral cochlear nucleus by intranuclear microstimulation. IEEE Trans. Rehab. Eng. 6: 391-399.

Mills SL and Massey SC 2000 A series of biotinylated tracers distinguishes three types of gap junction in retina. J. Neurosci. 20: 8629-8636.

Miyashita-Lin EM, et al. 1999 Early neocortical regionalization in the absence of thalamixc innervation. Science 285: 906-909.

Peachey NS and Chow AY 1999 Subretinal implantation of semi-conductor-based photodiodes: pregress and challenges. J. Rehab. Res. Dev. 36: 371-376.

Rauschecker JP and Shannon RV 2002 Sending sound to the brain. Science 295: 1025-1029.

Roska T and Chua LO 1993 The CNN universal machine: an analogic array computer. IEEE Trans. Circuits and Systems II 40: 163-173.

Roska B, et al. 2000 Three levels of lateral inhibition: A space-time study of the retina of the tiger salamander. J. Neurosci. 20: 1941-1951.

Roska B and Werblin F 2001 Vertical interactions across ten parallel, stacked representations in the mammalian retina. Nature 410: 583-587.

Santos A, et al. 1997 Preservation of the inner retina in retinitis pigmentosa - a morphometric analysis. Arch. Ophthalmol. 115: 511-515. 
Sharma J, Angelucci A and Sur M 2000 Induction of visual orientation modules in auditory cortex. Nature 404: 841-847.

Shostak Y, Ding Y, Mavity-Hudson J and Casagrande VA 2002 Cortical synaptoc arrangements of the third visual pathway in three primate species: Macaca mulatta, Siamiri sciureus, and Aotus trivirgatus J. Neurosci. 22: 2885-2893.

Shouval HZ, et al. 2000 Structured long-range connections can provide a scaffold for orientation maps. J. Neurosci. 20: 1119-1128.

Smith RG 1992 NeuronC: a computational language for investigating functional architecture of neural circuits. J. Neurosci. Meth. 43: 83-108.

Stroemer RP, Kent TA and Hulsebosch CE 1995 Neocortical neural sprouting, synaptogenesis and behavioral recovery after neocortical infarction in rats. Stroke 26: 2135-2144.

Strettoi E, et al. 2002 Morphological and functional abnormalities in the inner retina of the rd/rd mouse. J. Neurosci. 22: 5492-5504.

Vaney DI and Weiler R 2000 Gap junctions in the eye: evidence for heteromeric, heterotypic and mixed-homotypic interactions. Brain Res. Rev. 32: 115-120.

von Melchner L, Pallas SL and Sur M 2000 Visual behavior mediated by retinal projections directed to the auditory pathway. Nature 404: 871-876.

Wyatt J and Rizzo J 1996 Ocular implants for the blind. IEEE Spectrum 33: 47-53.

Wyatt J and Rizzo J 1997 The prospects for a visual prosthesis. The Neuroscientist 3: 251262.

Xerri C, et al. 1998 Plasticity of primary somatosensory cortex paralleling sensorimotor skill recovery from stroke in adult monkeys. J. Neurophysiol. 79: 2119-2148.

Yagi T 1999 A prototype of a micro-electrode array for hybrid retinal implant. Invest. Ophth. Vis. Sci. 40: S734 (Abstract).

Zrenner E 2002 Will retinal implants restore vision? Science 295: 1022-1025.

Zrenner E, et al. 1999 Can subretinal microphotodiodes successfully replace degenerated photoreceptors. Vis. Res. 39: 2555-2567. 


\section{INTERNAL DISTRIBUTION}

$\begin{array}{ll}1 & \text { B. G. Beckerman } \\ 2-10 & \text { M. Beckerman } \\ 11 & \text { C. L. Britton, Jr. } \\ 12 & \text { W. L. Clements } \\ 13-17 & \text { E. Greenbaum } \\ 18 & \text { S. E. Jacobsen } \\ 19 & \text { N. A. Johnson } \\ 20 & \text { J. S. Payne } \\ 21 & \text { R. E. Textor } \\ 22 & \text { T. G. Thundat } \\ 23-27 & \text { D. Cole, U.S. Department of Energy, 19901 Germantown Road, SC-73, Rm J- } \\ & \text { 116, Mail Stop SC73, Germantown, MD 20874-1290 } \\ 28 & \text { TC Resource Center, 1099 COM, Rm S07, MS7615 } \\ 29 & \text { Y-12 Central File, Bldg. 9711-5, MS8169 }\end{array}$

\section{EXTERNAL DISTRIBUTION}

O. Auciello, Argonne National Laboratory, 9700 South Cass Avenue, Argonne, IL 60439

31 J. Carlisle, Argonne National Laboratory, 9700 South Cass Avenue, Argonne, IL 60439

32 J. George, Los Alamos National Laboratory, MS D454, Los Alamos, NM 87545

33 R. Greenberg, Second Sight, P.O. Box 905, Santa Clarita, CA 91380-9005

34 D. Gruen, Argonne National Laboratory, 9700 South Cass Avenue, Argonne, IL 60439

35 M. Humayun, University of Southern California, 1450 San Pablo Street, Bldg. DEI 3600, Los Angeles, CA 90033

36 J. Jessing, Sandia National Laboratories, IMEMs \& Advanced Radiation Hard, CMOS Technology, P.O. Box 5800, MS 1074, Albuquerque, NM 87111

37 P. Krulevitch, Lawrence Livermore National Laboratory, P.O. Box 808, L-222, Livermore, CA 94551

38 G. Lazzi, North Carolina State University, 1010 Main Campus Drive, EGRC Rm. 422, Raleigh, NC 27606

39 W. Liu, North Carolina State University, 1010 Main Campus Drive, EGRC Rm. 422, Raleigh, NC 27606

40 M. Maghribi, Lawrence Livermore National Laboratory, P.O. Box 808, L-223, Livermore, CA 94551

41 B. Mech, Second Sight, P.O. Box 905, Santa Clarita, CA 91380-9005

42 R. L. Myers, Sandia National Laboratories, P.O. Box 5800, MS 1074, Albuquerque, NM 87111

43 O. Murat, Sandia National Laboratories, P.O. Box 5800, MS 1074, Albuquerque, NM 87111 
44 R. G. Smith, Department of Neuroscience, 123 Anatomy-Chemistry Bldg., University of Pennsylvania, Philadelphia, PA 19104-6058

45 J. Weiland, University of Southern California, Los Angeles, CA 90089

46 K. Wessendorf, Sandia National Laboratories, P.O. Box 5800, MS 1074, Albuquerque, NM 87111

47 D. Zhou, Second Sight, P.O. Box 905, Santa Clarita, CA 91380-9005 